\title{
Colour Calibration for Colour Reproduction
}

\author{
P. Emmel, R.D. Hersch \\ Ecole Polytechnique Fédérale de Lausanne, Switzerland \\ Patrick.Emmel@epfl.ch,Rd.Hersch@epfl.ch
}

\begin{abstract}
Due to the proliferation of low-cost colour devices (digital colour cameras, scanners, printers etc.) during the last few years, colour calibration has become an important issue. Such devices should faithfully reproduce colour images, but experience shows they don't. Among the main reasons, we note the diversity of acquisition, display and printing technologies which makes standardization difficult. Each device has a different gamut, i.e. a different set of colours that it can acquire or reproduce. Furthermore, the characteristics of the devices often vary with time. Hence a calibration procedure is unavoidable for high quality colour reproduction.
\end{abstract}

We give a brief overview of the colour calibration standard proposed by the International Colour Consortium (ICC), and then we present new approaches based on colour prediction models. Instead of using a large number of colour samples which must be produced and measured, only a few characteristic parameters are measured. The data corresponding to all samples are computed by a colour prediction software. This allows a quick recalibration when, for instance, the paper or the ink cartridge of a printer is changed.

\section{INTRODUCTION}

Today's digital colour reproduction market is characterized by two main classes of colour printing applications: desktop reproduction and high-speed on demand printing. Desktop reproduction enables individual users to directly create and ouput their documents. For them, there is a large offer of low-cost, high-quality, low throughput printers (HP, Canon, Epson, etc.). High-speed on demand printing machines are generally operated by print shops who are used to work with offset machines. These professional print shops have high printing volumes and require therefore flexible highthroughput machines.

The main printing technologies are ink-jet and colour laser printing with either solid toners (Canon CLC, Tektronix Phaser 550) or special inks (Indigo). For the desktop, dye sublimation and wax transfer technologies are also used. These technologies, especially inkjet and laser printing, are being constantly improved. Higher quality and lower priced devices are proposed both for desktop reproduction and for high-speed on demand printing.
Higher quality colour reproduction is obtained by enlarging the colour gamut. This can be attained by printing with more than the traditional process colours Cyan, Magenta, Yellow and Black (hexachrome printing for example). Maintaining a high degree of colour fidelity throughout the colour reproduction process requires the appropriate calibration of input devices (scanners), display devices (monitors) and output devices (printers).

In this paper, we will focus our attention on current approaches for calibrated colour reproduction and present a new approach based on colour prediction models.

\section{CALIBRATED COLOUR REPRODUCTION}

The International Colour Consortium, founded by several computer and peripheral equipment manufacturers (Adobe, AgfaGevaert, Apple, Kodak, Microsoft, Silicon Graphics, Sun Microsystems, Taligent) and by FOGRA (the German graphic arts research institute), has created a standard for describing the device profiles of input, display (preview) and output devices [14].

The input device profiles for scanners and digital cameras relate scanned R,G,B data to a device-independent profile connection space, either CIE-XYZ or CIE-LAB (these colour spaces are well described in [20]). The input model (Fig. 1) consists of non-linear tone reproduction curves (TRC) for mapping device red green and blue values (device RGB) to linear red green and blue values (linear RGB) and of a linear $3 \times 3$ transformation for mapping the linear red green and blue values to the connection space CIE-XYZ values. Equation (1) illustrates the transformation between the input device RGB space and the CIE-XYZ connection space.

Display devices are considered to be either colour CRT or colour liquid cristal displays. RGB display profiles relate values in the device independent space back into displayed RGB data. The display model is, therefore, the inverse of the input model, and it involves the inverse transformation as shown in equation (2). The profiles are characterized by each of the RGB channel's tone reproduction curve (redTRC, greenTRC, blueTRC) and the CIE$\mathrm{XYZ}$ values of their respective phosphors (or filters). These values are used to build the $3 \times 3$ matrix of equation (2) (note that these values are not the same as in equation (1)).

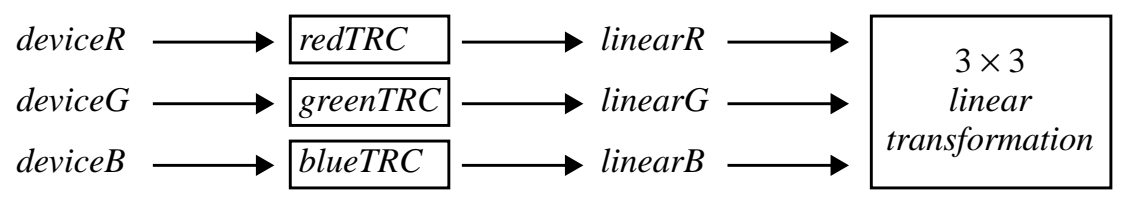

Figure 1. ICC input device calibration profile.

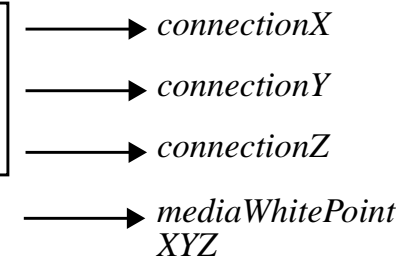

$X Y Z$ 
Colour printing on paper is a strongly non-linear process. Professional output device calibration systems are generally based on 3dimensional mapping tables. A 3D mapping table is constructed by printing combinations of all 3 or 4 output colours of the printer in question (cyan, magenta, yellow and black) at given output intensity intervals and by measuring the ouput samples as CIE-XYZ tristimulus values (see [12]). The set of measured samples enables establishing a correspondance between CIE-XYZ and output CMYK values. The set of measured samples in CIE-XYZ can be tetrahedrized. Within each tetrahedron, linear interpolation can be applied in order to compute for any new CIE-XYZ value its corresponding CMYK value (Fig. 2).

$$
\begin{aligned}
& \int \operatorname{lin} R=\operatorname{redTRC}[\text { device } R] \\
& \operatorname{lin} G=\text { greenTRC }[\text { device } G] \\
& \operatorname{lin} B=\text { blueTRC}[\text { device } B] \\
& \left\{\begin{array}{l}
\operatorname{con} X \\
\operatorname{con} Y \\
\operatorname{con} Z
\end{array}\right]=\left[\begin{array}{lll}
\text { redColX } & \text { green } \operatorname{Col} X & \text { blueColX } \\
\text { redColY } & \text { green } \operatorname{Col} Y & \text { blueColY } \\
\text { redColZ } & \text { green } \operatorname{Col} Z & \text { blueColZ }
\end{array}\right]\left[\begin{array}{c}
\operatorname{lin} R \\
\operatorname{lin} G \\
\operatorname{lin} B
\end{array}\right]
\end{aligned}
$$

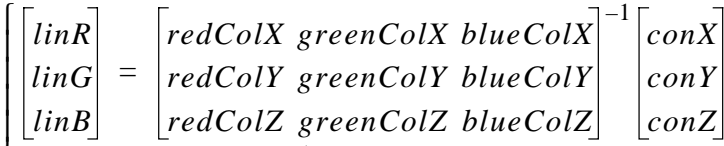

$$
\begin{aligned}
& \left\{\text { device } R=\operatorname{redTR} C^{-1}[\operatorname{lin} R]\right. \\
& \text { device } G=\text { greenTRC } C^{-1}[\operatorname{lin} G] \\
& \text { device } B=\text { blue } T R C^{-1}[\operatorname{lin} B]
\end{aligned}
$$

The ICC output device profiles characterizing the transformation from device-independent connection space (CIE-XYZ) to output space (CMYK or output RGB) is shown in (Fig. 3). It is composed of a linear transformation ( $3 \times 3$ matrix), a 1D look-up table associated to each channel, a multidimensional look-up table and finally a 1D look-up table associated to each output channel. The dimensionality of the multidimensional look-up table is given by the number of input channels (generally 3 ). Each multidimensional look-up table entry contains as many values as output channels. Therefore, the profile characterizing a CIE-XYZ to CMYK conversion will contain a 3D look-up table, where the $\mathrm{X}, \mathrm{Y}, \mathrm{Z}$ values are indices to access the table and where each table entry contains either a Null value (out of gamut colour) or a valid Cyan Magenta Yellow and Black value. One way of generating the 3D look-up table consists of computing the CMYK values of corresponding CIE-XYZ table entries (for a 3D grid in CIE-XYZ) by interpolating them in the tetrahedrized sample space described above.
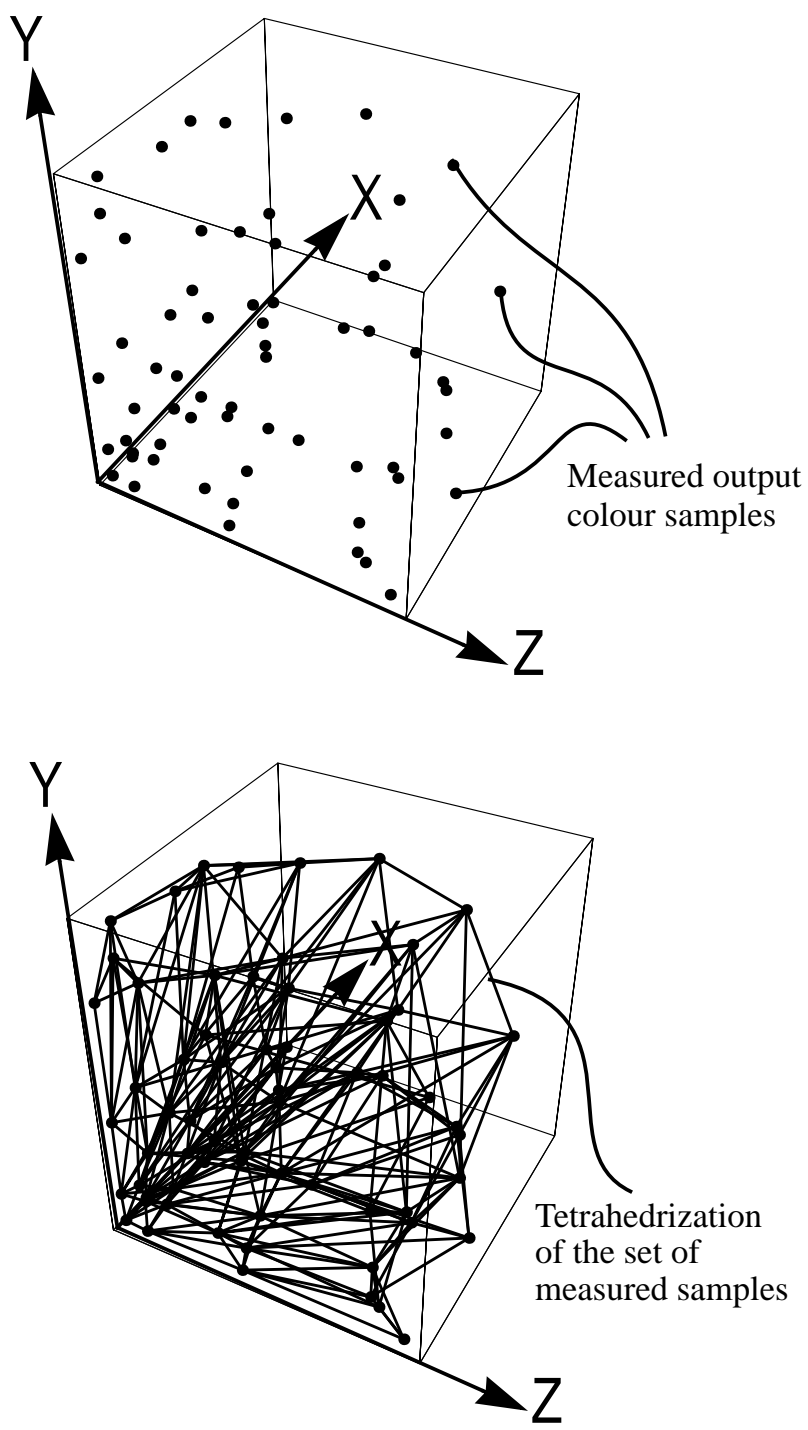

Figure 2. Tetrahedrization of the CIE-XYZ space based on the measured output samples.

In addition to profiles for input, display and output devices, ICC profiles incorporate information about how measurement were made ( $0 / 45$ degrees illumination geometry or diffuse illumination) and what the standard illuminants are (D50, D65, A, etc.). It also incorporates the viewing conditions (absolute XYZ of illuminant and surround in $\mathrm{cd} / \mathrm{m}^{2}$ ) and printing conditions (screen angle, frequency and spot shape).

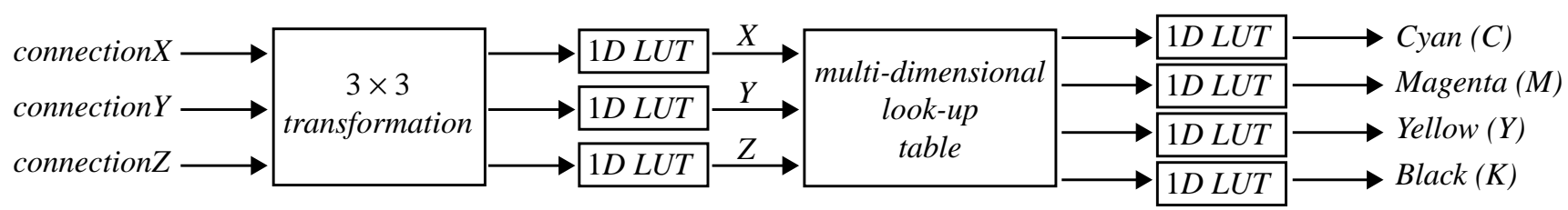

Figure 3. ICC output device calibrationprofile. 
Generally, the values given in the profile connection space (CIE$\mathrm{XYZ}$ ) are based on relative colorimetry, meaning that they are measured relative to a given illuminant, for example D50. When such values have to be displayed, respectively printed, the reference white $\mathrm{CIE}-\mathrm{XYZ}_{\mathrm{i}}$ is transformed to media white (display white, respectively paper white). In that case, in order to obtain the printable tri-stimulus values $\mathrm{CIE}-\mathrm{XYZ}_{\mathrm{a}}$, the connection space $\mathrm{CIE}$ $\mathrm{XYZ}_{\mathrm{r}}$ values have to be rescaled so as to match the new media white $\left(\mathrm{XYZ}_{\mathrm{mw}}\right)$.

$$
\begin{aligned}
& X_{a}=\left(\frac{X_{m w}}{X_{i}}\right) X_{r} \\
& Y_{a}=\left(\frac{Y_{m w}}{Y_{i}}\right) Y_{r} \\
& Z_{a}=\left(\frac{Z_{m w}}{Z_{i}}\right) Z_{r}
\end{aligned}
$$

In general, input devices and display devices have a larger gamut than output devices [7]. The mapping of the larger gamut onto the smaller must also be part of the calibration profile since a different rendering intent induces a different gamut mapping method (an overview of current gamut mapping methods is given in [3] and [4]). Therefore, the ICC profile format supports the following four rendering intents: perceptual, relative colorimetric, saturation and absolute colorimetric. Perceptual rendering means rendering on the output device so that input picture and output picture are perceptually similar, even if the measured colours vary considerably. Rendering by maintaining the relative colorimetry requires the adaptation of the colours to the new reference white as shown above. Saturation rendering consists in producing well saturated output images, keeping the hue as constant as possible. Absolute colorimetric rendering requires that all colours within the connection space are referenced with respect to an illuminant with known luminance $\left(\mathrm{cd} / \mathrm{m}^{2}\right)$ and that exactly the same colours are reproduced on the output device, independently of the media white point. Out of gamut colours are not reproduced.

The ICC standard profile format therefore provides the framework within which device manufacturers and application program producers may support input, display and output device profiles. It is up to the colour management systems integrated into operating systems (for example the ColorSync 3.0 for the MacIntosh operating system) to make use of the device profiles and reproduce the colour images accordingly.

The ICC standard however does not specify how to generate input, display and output device profile parameters. Generation of profile parameters appropriate for the different devices (scanners, displays, colour printers) remains part of the know-how of each of the contributing industries.

\section{NEW APPROACHES}

Good colour calibration requires printing and measuring about 1000 colour samples [11] to build the 3D mapping table. This large number makes calibration cumbersome, although new measuring devices accelerate the procedure [9]. A different approach consists of measuring only a few physical parameters and computing, thanks to a colour prediction model, the values which must be put into the various look-up tables of the calibration profile.
Most colour prediction models for printed halftoned samples derive from Neugebauer's model [18]. In the case of CMY printing, it requires measuring 8 primary colours which correspond to the paper white, the three solid ink colours cyan, magenta, yellow, and their superpositions red, green, blue and black. Let us denote $R_{w}(\lambda)$ the reflection spectrum of the paper, $R_{c}(\lambda), R_{m}(\lambda), R_{y}(\lambda)$ the reflection spectra of cyan, magenta, yellow, $R_{r}(\lambda), R_{g}(\lambda)$, $R_{b}(\lambda)$ the reflection spectra of red, green, blue, and $R_{k}(\lambda)$ the reflection spectrum of black (cyan over magenta over yellow). According to Neugebauer, the reflection spectrum $R(\lambda)$ of a halftoned sample is given by:

$$
\begin{aligned}
R(\lambda)= & (1-c)(1-m)(1-y) \cdot R_{w}(\lambda) \\
& +c(1-m)(1-y) \cdot R_{c}(\lambda) \\
& +(1-c) m(1-y) \cdot R_{m}(\lambda) \\
& +(1-c)(1-m) y \cdot R_{y}(\lambda) \\
& +(1-c) m y \cdot R_{r}(\lambda) \\
& +c(1-m) y \cdot R_{g}(\lambda) \\
& +c m(1-y) \cdot R_{b}(\lambda) \\
& +c m y \cdot R_{k}(\lambda)
\end{aligned}
$$

where $c$ is the fraction of area covered by the cyan ink, $m$ is the fraction of area covered by the magenta ink, and $y$ is the fraction of area covered by the yellow ink. This relation was established for traditional offset printing using three standard screens rotated at 30 (see [13]). In this particular case, the dot locations for the inks are statistically independent. Note that Neugebauer's model is easily generalized for $N$ inks [2].

Advanced Neugebauer-models take also into account surface reflection, internal reflection and light scattering in the paper. Their prediction accuracy was investigated by Kang [15] who concluded that improvements can be gained by measuring also intermediate points. Further studies by Balasubramanian [2] showed that good accuracy is obtained when about 500 samples are measured. Nevertheless, an acceptable trade-off between accuracy and number of measurements requires only about 100 samples.

Traditional Neugebauer models fail for two main reasons. First, when the screening method does not guarantee the randomness of the position of the ink dots. To circumvent this problem, the relative area covered by each ink combination is computed, and used as weighting factor in the Neugebauer equation. Second, since light scattering is a complex phenomenon, it is modelled with an empirical parameter called Yule-Nielsen factor [16] whose estimation requires many measurements. This is the Achilles' heel of all Neugebauer-based prediction methods. Other classical models, as for instance the Kubelka-Munk theory [17] which is also widely used in industry, handle light scattering much better. Unfortunately, they apply only to samples without halftones.

Recently several studies investigated light scattering in halftoned prints (see [1], [10] and [19]) leading to interesting new results and prediction models. They allow a new insight into the phenomenon, but their mathematical treatment is often very complex which makes these prediction models impractical.

The use of a new mathematical formulation based on matrices allows to express in a simple way the Kubelka-Munk reflection theory [5]. Furthermore, this formulation allows the merging of the Neugebauer model and the Kubelka-Munk model [6]. The complexity induced by light scattering is nicely reduced to boundary 
conditions of a linear matrix differential equation of the first order. This kind of equation is well known by mathematicians, and there are good algorithms to compute the solution [8]. Hence, a whole calibration profile can be computed using the physical data describing the inks and the paper.

This model requires measuring the refractive index of the surface, the reflection spectrum of the paper and the absorption- and scattering-spectra of the inks. In order to avoid the use of sophisticated physical instruments, a reduced set of "well designed" samples is measured. By inverting the equations of the colour prediction model, the physical parameters can be deduced and used to generate the calibration profile.

\section{CONCLUSION}

The calibration of scanning, previewing and printing paves the way to high-quality colour reproduction. Nevertheless, due to variations in the behaviour of the scanners, displays and printers (inks, paper) over time, frequent recalibration is necessary. Dynamic recalibration will become possible when special additional colorimetric sensors will be included in scanners, monitors and printers. Their data may be used by a colour prediction model to dynamically recompute new profiles when reproduction conditions change.

\section{REFERENCES}

[1] Arney J.S., Wu T., Blehm C., "Modeling the Yule Nielsen Effect on Color Halftones," Journal of Imaging Science and Technology, Vol. 42, No. 4, July/August 1998, pp. 335-340.

[2] Balasubramanian R., "Optimization of the spectral Neugebauer model for printer characterization," Journal of Electronic Imaging, Vol. 8(2), April 1999, pages 156-166.

[3] Commission International de l'Eclairage, web page of the CIE technical committee 8-03 on Gamut Mapping, http://www. colour.org/tc8-03/

[4] Ebner F., Fairchild M.D., "Gamut Mapping from Below: Finding Minimum Perceptual Distances for Colors Outside the Gamut Volume," Color Research and Application, Vol. 22, No. 6, December 1997, pages 402-413.

[5] Emmel P., Hersch R.D., "Towards a Color Prediction Model for Printed Patches," IEEE Computer Graphics \& Applications, Vol. 19, No. 4, July/August 1999, pp. 54-60.

[6] Emmel P., Hersch R.D., "A Model for Colour Prediction of Halftoned Samples Incorporating Light Scattering and Ink
Spreading," Proceedings of the $7^{\text {th }} I S \& T / S I D$ Color Imaging Conference, November 16-19, 1999, Scottsdale, Arizona, USA.

[7] Fairchild M.D., Color Appearance Models, Addison Wesley, 1998, pages 355-359.

[8] Golub G.H., Van Loan C.F., Matrix Computations, Third Edition, Johns Hopkins University Press, Baltimore, USA, pages 572-578.

[9] GretagMacbeth products: Spectrolino and Spectroscan, http: / / www.gretagmacbeth.com/lino.htm

[10] Gustavson S., Dot Gain in Colour Halftones, PhD thesis No. 492, Linköping University, Sweden, September 1997.

[11] Heuberger K.J., Jing Z.M., Persiev S., "Color Transformations and Look-Up Tables," 1992 TAGA/ISCC Proceedings, Vol. 2, pages 863-881.

[12] Hung P.-C., "Colorimetric calibration in electronic imaging devices using a look-up-table model and interpolations," Journal of Electronic Imaging, Vol. 2(1), January 1993, pages 53-61.

[13] Hunt R.W.G., The Reproduction of Colour in Photography, Printing \& Television, Fountain Press, Tolworth, England, 1987, page 534.

[14] ICC Profile Format Specification, Version ICC.1:1998-09, International Color Consortium, September, 1998 (see http: //www. color.org/).

[15] Kang H.R., "Applications of color mixing models to electronic printing," Journal of Electronic Imaging, Vol. 3(3), July 1994, pages 276-287.

[16] Kang H.R., Color Technology for Electronic Imaging Devices, SPIE Optical Engineering Press, Bellingham, Washington, USA, 1997, pages 43-45.

[17] Kubelka P., Munk F., "Ein Beitrag zur Optik der Farbanstriche," Zeitschrift für technische Physik, Vol. 12, 1931, pp. 593-601.

[18] Neugebauer H.E.J., "Die theoretischen Grundlagen des Mehrfarbenbuchdrucks," Zeitschrift für wissenschaftliche Photographie, Vol. 36(4), 1937, pages 73-89.

[19] Rogers G.L., "Effect of Light Scatter on Halftone Color," Journal of the Optical Society of America A, Vol. 15, No. 7, July 1998, pages 1813-1821.

[20] Wyszecki G., Stiles W.S., Color Science: Concepts and Methods, Quantitative Data and Formulae, Second Edition, John Wiley \& Sons, New York, 1982, pages 157-169. 